\title{
Parametrization and stress-energy-momentum tensors in metric field theories
}

\author{
Marco Castrillón López ${ }^{1}$, Mark J Gotay ${ }^{2}$ and Jerrold E Marsden ${ }^{3}$ \\ ${ }^{1}$ Departamento de Geometría y Topología, Facultad de Ciencias Matemáticas, \\ Universidad Complutense de Madrid, 28040 Madrid, Spain \\ 2 Department of Mathematics, University of Hawai 'i, Honolulu, HI 96822, USA \\ ${ }^{3}$ Control and Dynamical Systems 107-81, California Institute of Technology, Pasadena, \\ CA 91125, USA
}

Received 13 December 2007

Published 11 August 2008

Online at stacks.iop.org/JPhysA/41/344002

\begin{abstract}
We give an exposition of the 1972 parametrization method of Kuchař in the context of the multisymplectic approach to field theory. The purpose of the formalism developed here is to make any classical field theory, containing a metric as a sole background field, generally covariant (that is, parametrized, with the spacetime diffeomorphism group as a symmetry group) as well as fully dynamic. This is accomplished by introducing certain 'covariance fields' as genuine dynamic fields. As we shall see, the multimomenta conjugate to these new fields form the Piola-Kirchhoff version of the stress-energy-momentum tensor field, and their Euler-Lagrange equations are vacuously satisfied. Thus, these fields have no additional physical content; they serve only to provide an efficient means of parametrizing the theory. Our results are illustrated with two examples, namely an electromagnetic field and a Klein-Gordon vector field, both on a background spacetime.
\end{abstract}

PACS number: $03.50 .-\mathrm{Z}$

\section{Introduction}

When one is dealing with classical field theories on a spacetime, the metric may appear as a given background field or it may be a genuine dynamic field satisfying the Einstein equations. The latter theories are often generally covariant, with the spacetime diffeomorphism group as a symmetry group, but the former often are considered to have only the isometry group of the metric as a symmetry group. However, Kuchař (1973) (see also Isham and Kuchař (1985)) indicated how theories with a background metric can be parametrized, that is, considered as theories that are fully covariant, if one introduces the diffeomorphisms themselves as dynamic fields. The goal of this paper is to develop this idea in the context of multisymplectic classical field theory and to make connections with stress-energy-momentum (SEM) tensors. As 
we shall see, the multimomenta conjugate to these new covariance fields form, to borrow a phrase from elasticity theory, the Piola-Kirchhoff version of the SEM tensor, and their Euler-Lagrange equations are vacuously satisfied by virtue of the fact that the SEM tensor is covariantly conserved. Thus these fields have no physical content; they serve only to provide an efficient way of parametrizing a field theory. Nonetheless, the resulting generally covariant field theory has several attractive features, the chief among which is that it is fully dynamicall fields satisfy Euler-Lagrange equations. Structurally, such theories are much simpler to analyze than those with absolute objects or noncovariant elements.

We emphasize that the results of this paper are for those field theories whose Lagrangians are built from dynamic matter or other fields and a non-dynamic background metric. One of our motivations was to find a way to treat background fields and dynamic fields in a unified way in the context of the adjoint formalism. Many of the ideas are applicable to a wider range of field theories, as Kuchař (1973) already indicates, but in this paper we confine ourselves to this important class. The general case is presented in Gotay and Marsden (2008b) along with a more detailed discussion of parametrization theory and related topics.

\section{The covariance construction}

Suppose that we have a metric field theory in which the metric is an 'absolute object' in the sense of Anderson (1967) (or a 'structural field' in the sense of Post (2007)). For instance, one might consider a dynamic electromagnetic field propagating on a Schwarzschild spacetime. Such a theory is not generally covariant, because the spacetime is fixed, and not all fields are on equal footing, as the electromagnetic field is dynamic while the gravitational field is not. A somewhat different example is provided by Nordstrøm's theory of gravity (see section 17.6 of Misner et al (1973)), which is set against a Minkowskian background.

In this section we explain how to take such a system and construct from it an equivalent field theory that achieves the following goals:

(I) the new field theory is generally covariant;

(II) all fields in the new field theory are dynamic.

This 'covariance construction' is an extension and refinement of the parametrization procedure introduced by Kuchař (1973).

As usual for a first-order classical field theory, we start with a bundle $Y \rightarrow X$ whose sections, denoted as $\phi$, are the fields under consideration. The dimension of $X$ is taken to be $n+1$, and we suppose that $X$ is oriented. Let

$$
\mathcal{L}: J^{1} Y \rightarrow \Lambda^{n+1} X
$$

be a Lagrangian density for this field theory, where $J^{1} Y$ is the first jet bundle of $Y$ and $\Lambda^{n+1} X$ is the space of top forms on $X$. Loosely following the notation of Gotay and Marsden (1992) or Gotay and Marsden (2008a), we write coordinates for $J^{1} Y$ as $\left(x^{\mu}, y^{A}, y^{A}{ }_{\mu}\right)$. In addition, in coordinates, we shall write

$$
\mathcal{L}=L\left(x^{\mu}, y^{A}, y^{A}{ }_{\mu}\right) d^{n+1} x .
$$

Evaluated on the first jet prolongation of a section $\phi$, the Lagrangian becomes a function of $\left(x^{\mu}, \phi^{A}, \phi^{A}{ }_{, \mu}\right)$; we shall abbreviate this when convenient and simply write $\mathcal{L}\left(j^{1} \phi\right)$. We assume that the fields $\phi$ are dynamic.

Example. We will intersperse the example of electromagnetism throughout the paper to illustrate our results. Then $Y$ is the cotangent bundle of the four-dimensional spacetime $X$, 
sections of which are electromagnetic potentials $A$. The corresponding Lagrangian is written below.

Suppose that the spacetime $X$ comes equipped with a fixed, background metric $g$. The obvious first step in attaining general covariance is to allow $g$ to vary; thus the metric will now be regarded as a genuine field $G$ on $X$. (When the metric is regarded as a variable, we denote it by $G$, and when we want to revert to its fixed value we use $g$.) So we are led to view the Lagrangian density as a map

$$
\mathcal{L}: J^{1} Y \times \operatorname{Lor}(X) \rightarrow \Lambda^{n+1} X
$$

where $\operatorname{Lor}(X)$ is the bundle whose sections are Lorentz metrics on $X$. We correspondingly write $\mathcal{L}\left(j^{1} \phi ; G\right)$; the semicolon is used to separate the dynamic from the nondynamic fields. (We emphasize that $G$ being variable does not mean that it is dynamic; we discuss this point momentarily.) Note that we have tacitly assumed that the dependence of $\mathcal{L}$ on the metric is pointwise - that is, we have non-derivative coupling. (The more general case of derivative coupling will be considered in section 5. In any event, we remark that derivatively-coupled theories are considered by many to be pathological.)

Example. The electromagnetic Lagrangian density

$$
\mathcal{L}: J^{1}\left(T^{*} X\right) \times \operatorname{Lor}(X) \rightarrow \Lambda^{4} X
$$

is

$$
\mathcal{L}\left(j^{1} A ; G\right)=-\frac{1}{4} G^{\mu \alpha} G^{\nu \beta} F_{\alpha \beta} F_{\mu \nu} \sqrt{-G} \mathrm{~d}^{4} x
$$

where $F_{\mu \nu}=A_{\nu, \mu}-A_{\mu, \nu}$.

Next, assume that the given Lagrangian density $\mathcal{L}$ has the following (eminently reasonable) covariance property for a diffeomorphism $\sigma: X \rightarrow X$ :

$$
\sigma_{*}\left(\mathcal{L}\left(j^{1} \phi ; G\right)\right)=\mathcal{L}\left(j^{1}\left(\sigma_{Y}(\phi)\right) ; \sigma_{*} G\right)
$$

where we assume that one way to lift the spacetime diffeomorphism $\sigma$ to a bundle automorphism $\sigma_{Y}$ of $Y$ has been chosen.

Example. For the electromagnetic 1-form potential $A$, we take the lift to be push-forward on the fiber, which makes it obvious that (2.2) holds in this case.

When condition (2.2) holds, we say that the theory is generally covariant, i.e., the Lagrangian density is Diff $(X)$-equivariant. Thus we have accomplished objective (I). However, the reader may well remark that this was 'too easy', and would be quite right. The problem is that it is not clear how, or even if, $G$ can now be made dynamic. Certainly, $G$ cannot be taken to be variational unless one adds a source term to the Lagrangian density for $G$, for otherwise

$$
\frac{\partial L}{\partial G_{\mu \nu}}=\frac{\delta L}{\delta G_{\mu \nu}}=0
$$

as the metric non-derivatively couples to the other fields. But what should this source term be? If $G$ is gravity, we could use the Hilbert Lagrangian, but otherwise this is unclear.

The covariance field. The solution to our problem requires more subtlety. We will sidestep both the issues of making $g$ variable, and then making $G$ dynamic, in one fell swoop as follows. We introduce an entirely new field, the 'covariance field' into the theory. It will 'soak up' the arbitrariness in $G$, and will be dynamic. In this way we are able to generate a new generally 


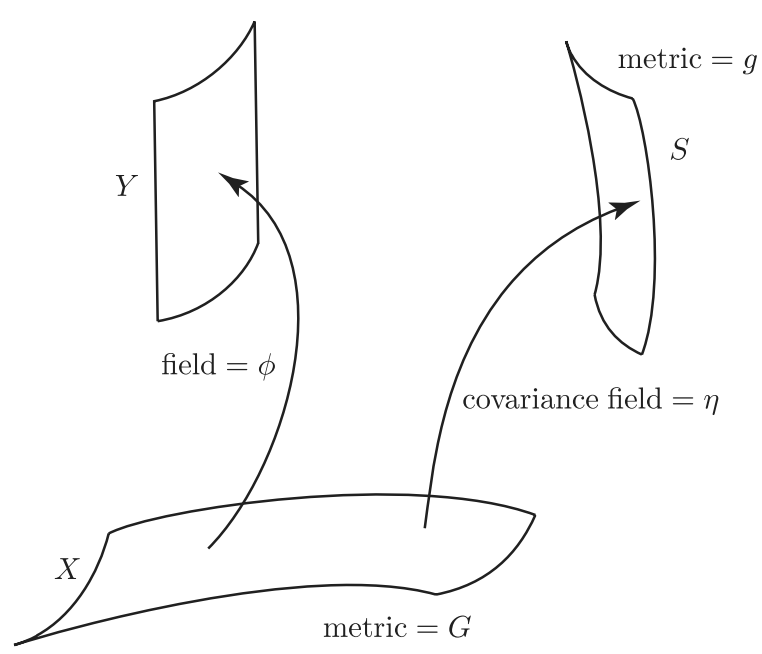

Figure 1. The general setup for the introduction of covariance fields.

covariant field theory, physically equivalent to the original one, in which all fields are dynamic. Here is the construction.

The key idea is to introduce a copy $(S, g)$ of spacetime into the fiber of the configuration bundle. Consider (oriented) diffeomorphisms $\eta: X \rightarrow S$, thought of as sections of the bundle $S \times X \rightarrow X$. We regard the diffeomorphisms $\eta$ as new fields and correspondingly replace the configuration bundle by $\widetilde{Y}=Y \times_{X}(S \times X) \rightarrow X$. Next, modify $\mathcal{L}$ to get the new Lagrangian $\widetilde{\mathcal{L}}$ defined on $J^{1} \widetilde{Y}$ :

$$
\widetilde{\mathcal{L}}\left(j^{1} \phi, j^{1} \eta\right)=\mathcal{L}\left(j^{1} \phi ; \eta^{*} g\right) .
$$

Thus, we obtain a modified field theory with the underlying bundle $\widetilde{Y}$. The general setup is shown in figure 1.

Let coordinates on $S$ be denoted as $u^{a}$ and the associated jet coordinates be denoted as $u^{a}{ }_{\mu}$. Then, writing $\mathcal{L}=L \mathrm{~d}^{4} x$ and similarly for $\widetilde{\mathcal{L}}$, in coordinates equation (2.3) reads

$$
\widetilde{L}\left(x^{\mu}, y^{A}, y_{\mu}^{A}, u^{a}, u_{\mu}^{a}\right)=L\left(x^{\mu}, y^{A}, y_{\mu}^{A} ; G_{\mu \nu}\right),
$$

where, from the definition of pull back

$$
G_{\mu \nu}(x):=\left(\eta^{*} g\right)_{\mu \nu}(x)=\eta_{, \mu}^{a}(x) \eta_{, \nu}^{b}(x) g_{a b}(\eta(x))
$$

we obtain

$$
G_{\mu \nu}=u_{\mu}^{a} u^{b}{ }_{\nu} g_{a b} .
$$

From (2.4) one verifies that the Euler-Lagrange equations for the fields $\phi^{A}$ remain unchanged.

Example. For the electromagnetic field, our construction produces

$$
\tilde{\mathcal{L}}\left(j^{1} A, j^{1} \eta\right)=-\frac{1}{4} g^{a c} g^{b d} \kappa^{\mu}{ }_{c} \kappa^{\alpha}{ }_{a} \kappa^{\nu}{ }_{d} \kappa^{\beta}{ }_{b} F_{\mu \nu} F_{\alpha \beta} \sqrt{-g}\left(\operatorname{det} \eta_{*}\right) \mathrm{d}^{4} x
$$

where $\eta_{*}$ is the Jacobian of $\eta$ and $\kappa=\eta^{-1}$.

We pause to point out the salient features of our construction. First, the fixed metric $g$ on spacetime is no longer regarded as living on $X$, but rather on the copy $S$ of $X$ in the fiber of the configuration bundle $\widetilde{Y}$. So $g$ is no longer considered to be a field-it has been demoted to 
a mere geometric object on the fiber $S$. Second, the variable metric $G$ on $X$ is identified with $\eta^{*} g$, and thus acquires its variability from that of $\eta$. So $G$ also is no longer a field per se, but simply an abbreviation for the quantity $\eta^{*} g$. Finally, we gain a field $\eta$ which we allow to be dynamic; in the following subsection we will see that this imposes no restrictions on the theory at all.

The first key observation is that the modified theory is indeed generally covariant. To this end, recall that, as was explained earlier, given $\sigma \in \operatorname{Diff} X$, there is assumed to be a lift $\sigma_{Y}: Y \rightarrow Y$. For the trivial bundle $S \times X$, we define

$$
\begin{gathered}
\sigma_{S}: S \times X \rightarrow S \times X \\
(u, x) \mapsto(u, \sigma(x)) .
\end{gathered}
$$

Theorem 2.1. The Lagrangian density $\widetilde{\mathcal{L}}: J^{1}\left(Y \times_{X}(S \times X)\right) \rightarrow \Lambda^{n+1} X$ is $\operatorname{Diff}(X)$-equivariant, that is,

$$
\sigma_{*}\left(\widetilde{\mathcal{L}}\left(j^{1} \phi, j^{1} \eta\right)\right)=\widetilde{\mathcal{L}}\left(j^{1}\left(\sigma_{Y}(\phi)\right), j^{1}\left(\sigma_{S}(\eta)\right)\right) .
$$

Proof. This is an easy consequence of definitions (2.3) and (2.7), and the covariance assumption (2.2). Indeed

$$
\begin{aligned}
\widetilde{\mathcal{L}}\left(j^{1}\left(\sigma_{Y}(\phi)\right), j^{1}\left(\sigma_{S}(\eta)\right)\right) & =\mathcal{L}\left(j^{1}\left(\sigma_{Y}(\phi)\right) ;\left(\eta \circ \sigma^{-1}\right)^{*} g\right) \\
& =\mathcal{L}\left(j^{1}\left(\sigma_{Y}(\phi)\right) ;\left(\sigma^{-1}\right)^{*}\left(\eta^{*} g\right)\right) \\
& \left.=\sigma_{*}\left(\mathcal{L}\left(j^{1} \phi\right) ;\left(\eta^{*} g\right)\right)\right) \\
& =\sigma_{*}\left(\widetilde{\mathcal{L}}\left(j^{1} \phi, j^{1} \eta\right)\right) .
\end{aligned}
$$

Because of this property, we call $\eta$ the covariance field.

Example. From (2.6) it is clear that the modified electromagnetic theory is generally covariant.

\section{The dynamics of the covariance field}

Next we will show something remarkable: the Euler-Lagrange equation for the covariance field $\eta$ is vacuous. This is the main reason why, in the present context, we can introduce $\eta$ as a dynamic field with impunity, namely, its Euler-Lagrange equation does not add any new information to, or impose any restrictions upon, the system. Since, as we mentioned earlier, the Euler-Lagrange equations for the fields $\phi^{A}$ remain unaltered, we see that the parametrized system is physically equivalent to the original system.

First we compute the multimomenta conjugate to the field $\eta$ for the parametrized field theory with Lagrangian $\widetilde{\mathcal{L}}$. Recall that in the multisymplectic field theory, the multimomenta conjugate to the multivelocities $u^{A}{ }_{\mu}$ are defined by

$$
\rho_{a}{ }^{\mu}=\frac{\partial \widetilde{L}}{\partial u_{\mu}^{a}} .
$$

Using the chain rule together with relations (2.4) and (2.5), we find that

$$
\rho_{a}{ }^{\mu}=2 \frac{\partial L}{\partial G_{\mu \nu}} u_{\nu}^{b} g_{a b} .
$$

Recall from Gotay and Marsden (1992) that, as we have assumed that $G$ is the only nondynamic field, and does not derivatively couple to the other fields, the SEM tensor density for the original system with Lagrangian $L$ and metric $G$ is given by the Hilbert formula:

$$
\mathfrak{T}^{\mu \nu}=2 \frac{\delta L}{\delta G_{\mu \nu}}=2 \frac{\partial L}{\partial G_{\mu \nu}} .
$$


From (3.1) we conclude that the multimomenta conjugate to the covariance field $\eta$ are given by the (first) Piola-Kirchhoff SEM tensor density:

$$
\rho_{a}{ }^{\mu}=\mathfrak{T}^{\mu v} u^{b}{ }_{\nu} g_{a b} .
$$

This is a familiar object in elasticity theory (see, for instance, Marsden and Hughes (1983), p 135). Observe that $\rho_{a}{ }^{\mu}$ is a two-point tensor density: it has one leg $(a)$ in the spacetime $S$ in the fiber-analogous to the spatial representation in elasticity theory, and the other leg $(\mu)$ in the spacetime $X$ in the base-analogous to the material representation.

Now we compute the Euler-Lagrange equations for the $\eta^{a}$. These are

$$
\frac{\partial \tilde{L}}{\partial \eta^{a}}-\frac{\partial}{\partial x^{\mu}}\left(\frac{\partial \tilde{L}}{\partial \eta^{a}, \mu}\right)=0
$$

for $a=1, \ldots, \operatorname{dim} X$. Expanding the derivatives via the chain rule and using the same type of calculation as in the derivation of (3.1) to write the equations in terms of $L$ rather than $\tilde{L}$, the preceding equation becomes

$$
\frac{\partial L}{\partial G_{\mu \nu}} \eta_{, \mu}^{c} \eta^{d}, \nu \frac{\partial g_{c d}}{\partial u^{a}}-2 \frac{\partial}{\partial x^{\mu}}\left(\frac{\partial L}{\partial G_{\mu \nu}} \eta^{c}{ }_{, \nu} g_{a c}\right)=0 .
$$

Replacing $\partial L / \partial G_{\mu \nu}$ by (half of) $\mathfrak{T}^{\mu \nu}$, and differentiating using the product rule, we obtain $\mathfrak{T}^{\mu \nu} \eta^{c}{ }_{, \mu} \eta^{d}{ }_{, \nu} \frac{\partial g_{c d}}{\partial u^{a}}-2\left(\frac{\partial \mathfrak{T}^{\mu \nu}}{\partial x^{\mu}} \eta^{c}{ }_{, \nu} g_{a c}+\mathfrak{T}^{\mu \nu} \eta^{c}{ }_{, \mu \nu} g_{a c}+\mathfrak{T}^{\mu \nu} \eta^{c}{ }_{, \nu} \frac{\partial g_{a c}}{\partial u^{d}} \eta^{d}, \mu\right)=0$, for $a=1, \ldots, \operatorname{dim} X$.

Multiplying by the inverse matrix $g^{a b}$ one gets $\mathfrak{T}^{\mu \nu} \eta_{, \mu}^{c} \eta^{d}{ }_{, \nu} \frac{\partial g_{c d}}{\partial u^{a}} g^{a b}-2\left(\frac{\partial \mathfrak{T}^{\mu \nu}}{\partial x^{\mu}} \eta^{b}{ }_{, \nu}+\mathfrak{T}^{\mu \nu} \eta_{, \mu \nu}^{b}+\mathfrak{T}^{\mu \nu} \eta^{c}{ }_{, \nu} \eta^{d}, \mu \frac{\partial g_{a c}}{\partial u^{d}} g^{a b}\right)=0$,

for $b=1, \ldots, \operatorname{dim} X$. And now, we multiply by $\kappa^{\rho}{ }_{b}$, the inverse matrix of the Jacobian $\eta^{b}, v$ $\mathfrak{T}^{\mu \nu} \eta^{c}{ }_{, \mu} \eta^{d}{ }_{, \nu} \frac{\partial g_{c d}}{\partial u^{a}} g^{a b} \kappa^{\rho}{ }_{b}-2\left(\frac{\partial \mathfrak{T}^{\mu \rho}}{\partial x^{\mu}}+\mathfrak{T}^{\mu \nu} \eta^{b}{ }_{, \mu \nu} \kappa^{\rho}{ }_{b}+\mathfrak{T}^{\mu \nu} \eta^{c}{ }_{, \nu} \eta^{d}{ }_{, \mu} \frac{\partial g_{a c}}{\partial u^{d}} g^{a b} \kappa^{\rho}{ }_{b}\right)=0$, for $v=1, \ldots, \operatorname{dim} X$. Taking into account the symmetry $\mathfrak{T}^{\mu \nu}=\mathfrak{T}^{\nu \mu}$, the preceding equation becomes

$\mathfrak{T}^{\mu \nu} \eta^{c}{ }_{, \mu} \eta^{d}{ }_{, \nu} \kappa^{\rho}{ }_{b}\left(\frac{\partial g_{c d}}{\partial u^{a}} g^{a b}-\frac{\partial g_{a d}}{\partial u^{c}} g^{a b}-\frac{\partial g_{a c}}{\partial u^{d}} g^{a b}\right)-2\left(\frac{\partial \mathfrak{T}^{\mu \rho}}{\partial x^{\mu}}+\mathfrak{T}^{\mu \nu} \eta^{b}{ }_{, \mu \nu} \kappa^{\rho}{ }_{b}\right)=0$.

Recalling the expression of the Christoffel symbols of the metric $g$, namely,

$$
\gamma_{c d}^{b}=\frac{1}{2} g^{a b}\left(\frac{\partial g_{a c}}{\partial u^{d}}+\frac{\partial g_{a d}}{\partial u^{c}}-\frac{\partial g_{c d}}{\partial u^{a}}\right)
$$

we obtain

$$
-2 \mathfrak{T}^{\mu \nu} \eta_{, \mu}^{c} \eta^{d}{ }_{, \nu} \gamma_{c d}^{b} \kappa^{\rho}{ }_{b}-2\left(\frac{\partial \mathfrak{T}^{\mu \rho}}{\partial x^{\mu}}+\mathfrak{T}^{\mu \nu} \eta_{, \mu \nu}^{b} \kappa_{b}^{\rho}\right)=0 .
$$

Finally, recall how the Christoffel symbols $\gamma_{c d}^{b}$ for $g$ and the symbols $\Gamma_{\mu \nu}^{\rho}$ for $G=\eta^{*} g$ are related:

$$
\Gamma_{\mu \nu}^{\rho}=\frac{\partial^{2} u^{b}}{\partial x^{\mu} \partial x^{\nu}} \frac{\partial x^{\rho}}{\partial u^{b}}+\frac{\partial u^{c}}{\partial x^{\mu}} \frac{\partial u^{d}}{\partial x^{\nu}} \gamma_{c d}^{b} \frac{\partial x^{\rho}}{\partial u^{b}} .
$$

Using this in (3.3) gives

$$
-2\left(\frac{\partial \mathfrak{T}^{\mu \rho}}{\partial x^{\mu}}+\mathfrak{T}^{\mu \nu} \Gamma_{\mu \nu}^{\rho}\right)=0,
$$

6 
for $v=1, \ldots, \operatorname{dim} X$, which is exactly the vanishing of the covariant divergence of the tensor density $\mathfrak{T}^{\mu \nu}$. (Note that the coordinate expression for the covariant divergence of an ordinary rank-2 tensor field has an extra term that does not appear in the previous equation. This term is canceled by yet another term which arises from the fact that $\mathfrak{T}^{\mu \nu}$ is a tensor density, cf section 2.1 in Anderson (1967).)

Thus, we have proven the following basic result.

Theorem 3.1. The Euler-Lagrange equations for the covariance field $\eta$ are that the covariant divergence of the SEM tensor density $\mathfrak{T}^{\mu \nu}$ is zero.

It is known from proposition 5 in Gotay and Marsden (1992) that the SEM tensor is covariantly conserved when the metric $G$ is the only nondynamic field. Thus, in our context, the equation $\nabla_{\mu} \mathfrak{T}^{\mu \nu}=0$ is an identity, whence

Corollary 3.2. The Euler-Lagrange equations for the covariance field $\eta$ are vacuously satisfied.

Consequently the covariance field has no physical import. We are free to suppose $\eta$ is dynamic, and so we have accomplished goal (II): we have constructed a new field theory in which all fields are dynamic.

\section{The SEM tensor}

It is interesting to compare the SEM tensors for the original and parametrized systems. In Gotay and Marsden (1992) the SEM tensor density $\mathfrak{T}^{\mu}{ }_{v}$ is defined in terms of fluxes of the multimomentum map $J^{\mathcal{L}}$ associated with the action of the spacetime diffeomorphism group. We rapidly recount some of the basic ideas.

Consider the lift of an infinitesimal diffeomorphism $\xi \in \mathfrak{X}(X)$ to $Y$; it can be expressed as

$$
\xi_{Y}=\xi^{\mu} \frac{\partial}{\partial x^{\mu}}+\xi^{A} \frac{\partial}{\partial y^{A}}
$$

where we suppose that

$$
\xi^{A}=C^{A \rho_{1} \cdots \rho_{k}}{ }_{\nu} \xi^{\nu}{ }_{, \rho_{1} \cdots \rho_{k}}+\cdots+C^{A \rho}{ }_{\nu} \xi^{\nu}, \rho+C^{A}{ }_{\nu} \xi^{\nu}
$$

for some coefficients $C^{A \rho_{1} \cdots \rho_{k}}{ }_{\nu}, \ldots, C^{A \rho}{ }_{\nu}, C^{A}{ }_{\nu}$. The largest value of $k$ for which one of the top coefficients $C^{A \rho_{1} \dot{S} \rho_{k}}{ }_{\nu}$ is nonzero is the differential index of the field theory. We assume henceforth that the index $\leqslant 1$ - the most common and important case (e.g., when the fields are all tensor fields).

In this context, theorem 1 along with remark 4 of Gotay and Marsden (1992) shows that the SEM tensor density $\mathfrak{T}$ for a Lagrangian density $\mathcal{L}$ is uniquely determined by

$$
\int_{\Sigma} i_{\Sigma}^{*}\left(j^{1} \phi\right)^{*} J^{\mathcal{L}}\left(\xi_{Y}\right)=\int_{\Sigma} \mathfrak{T}^{\mu}{ }_{\nu}(\phi) \xi^{\nu} \mathrm{d}^{n} x_{\mu}
$$

for all vector fields $\xi$ on $X$ with compact support and all hypersurfaces $\Sigma$, where $i_{\Sigma}: \Sigma \rightarrow X$ is the inclusion. The multimomentum map $J^{\mathcal{L}}$ gives, roughly speaking, the flow of momentum and energy through spacetime; according to the quoted theorem, the fluxes of this flow across hypersurfaces are realized via the SEM tensor density.

Manipulation of (4.1) (see formula (3.12) of Gotay and Marsden (1992)) shows that $\mathfrak{T}$ is given by

$$
\mathfrak{T}_{\nu}^{\mu}=L \delta^{\mu}{ }_{\nu}-\frac{\partial L}{\partial \psi^{A}{ }_{, \mu}} \psi_{, \nu}^{A}+\frac{\partial L}{\partial \psi^{A}{ }_{, \mu}} C^{A}{ }_{\nu}+D_{\rho}\left(\frac{\partial L}{\partial \psi^{A}{ }_{, \rho}} C^{A \mu}{ }_{\nu}\right)
$$

where the summation extends over all fields $\psi^{A}$. 
We apply this to the newly parametrized theory. Note that if the index of the original theory is $\leqslant 1$, then that for the parametrized theory will be $\leqslant 1$ also. As well from (2.7) we see that the lift of $\xi$ to $S \times X$ is trivial:

$$
\xi^{a}=0
$$

that is, there are no terms in the $\partial / \partial u^{a}$ directions in $\xi \widetilde{Y}$. Thus the corresponding coefficients $C^{a \cdots}{ }_{v}$ all vanish. The SEM tensor for $\widetilde{\mathcal{L}}$ therefore reduces to

$$
\widetilde{\mathfrak{T}}^{\mu}{ }_{\nu}=\widetilde{L}{\delta^{\mu}}^{\mu}-\frac{\partial \widetilde{L}}{\partial \phi^{A}{ }_{, \mu}} \phi^{A}{ }_{, \nu}+\frac{\partial \widetilde{L}}{\partial \phi^{A}{ }_{, \mu}} C^{A}{ }_{\nu}+D_{\rho}\left(\frac{\partial \widetilde{L}}{\partial \phi^{A}{ }_{, \rho}} C^{A \mu}{ }_{\nu}\right)-\frac{\partial \widetilde{L}}{\partial \eta^{a}, \mu} \eta^{a}{ }_{, \nu} .
$$

On the other hand,

$$
\frac{\partial \widetilde{L}}{\partial \eta^{a}{ }_{, \mu}} \eta^{a}{ }_{, \nu}=2 \frac{\partial L}{\partial G_{\mu \rho}} \eta_{, \rho}^{b} g_{a b} \eta^{a}{ }_{, \nu}=2 \frac{\partial L}{\partial G_{\mu \rho}} G_{\rho \nu}
$$

and

$$
\frac{\partial \widetilde{L}}{\partial \phi^{A}, \mu}=\frac{\partial L}{\partial \phi^{A}, \mu},
$$

so that we can write

$$
\widetilde{\mathfrak{T}}^{\mu}{ }_{\nu}=L \delta^{\mu}{ }_{\nu}-\frac{\partial L}{\partial \phi^{A}, \mu} \phi^{A}{ }_{, \nu}+\frac{\partial L}{\partial \phi^{A}, \mu} C^{A}{ }_{\nu}+D_{\rho}\left(\frac{\partial L}{\partial \phi^{A}, \rho} C^{A \mu}{ }_{\nu}\right)-2 \frac{\partial L}{\partial G_{\mu \rho}} G_{\rho \nu} .
$$

But the first four terms on the rhs of this equation comprise the SEM tensor density of the original theory since $G_{\mu \nu}$ do not derivatively couple to the $\phi^{A}$ (cf equation (4.4) in Gotay and Marsden (1992)). Thus the SEM tensor densities of the original and parametrized systems are related according to

\section{Proposition 4.1.}

$$
\widetilde{\mathfrak{T}}^{\mu}{ }_{\nu}=\mathfrak{T}^{\mu}{ }_{\nu}-2 \frac{\partial L}{\partial G_{\mu \rho}} G_{\rho \nu} .
$$

But then $\widetilde{\mathfrak{T}}^{\mu}{ }_{v}=0$ on shell by the Hilbert formula (3.2). Therefore, we explicitly see that the SEM tensor density for the fully covariant, fully dynamic modified theory vanishes. One can also obtain this result directly by applying the generalized Hilbert formula (3.13) in Gotay and Marsden (1992) to the parametrized theory, since it is fully dynamic.

Example. In the case of electromagnetism, one may compute directly from (2.6) that $\widetilde{\mathfrak{T}}^{\mu}{ }_{v}=0$. One could also compute from (2.1) that

$$
\mathfrak{T}^{\mu}{ }_{\nu}=-\left(\frac{1}{4} \delta^{\mu}{ }_{\nu} F_{\alpha \beta} F^{\alpha \beta}+F^{\alpha \mu} F_{\nu \alpha}\right) \sqrt{-G}=2 \frac{\partial L}{\partial G_{\mu \rho}} G_{\rho \nu} .
$$

\section{Derivative couplings}

Here we briefly consider the situation, although perhaps exotic, when the metric derivatively couples to the other fields. For simplicity, however, we suppose the theory remains first order. So the Lagrangian density is taken to be a map

$$
\mathcal{L}: J^{1}\left(Y \times_{X} \operatorname{Lor}(X)\right) \rightarrow \Lambda^{n+1} X .
$$

As before, modify $\mathcal{L}$ to get the new Lagrangian $\widetilde{\mathcal{L}}$ defined on $J^{2} \widetilde{Y}$ :

$$
\widetilde{\mathcal{L}}\left(j^{1} \phi, j^{2} \eta\right)=\mathcal{L}\left(j^{1} \phi ; j^{1}\left(\eta^{*} g\right)\right) .
$$


(Since $\eta^{*} g$ depends upon the first derivatives of $\eta, j^{1}\left(\eta^{*} g\right)$ will depend upon its second derivatives. Thus, we obtain a modified second-order field theory with the underlying bundle $\widetilde{Y}$.) The discussion proceeds as in the above, with only obvious changes. In particular, if $\mathcal{L}$ is $\operatorname{Diff}(X)$-covariant, then so is $\widetilde{\mathcal{L}}$.

Example. As a simple illustration of a derivatively coupled theory, consider a vector meson with mass $m$. Then $Y$ is the tangent bundle of spacetime and its sections $\phi^{\mu}$ are Klein-Gordon vector fields. The Lagrangian density is the map

$$
\mathcal{L}: J^{1}\left(T X \times_{X} \operatorname{Lor}(X)\right) \rightarrow \Lambda^{4} X
$$

defined by

$$
\mathcal{L}\left(j^{1} \phi ; j^{1} G\right)=\frac{1}{2} G_{\sigma \rho}\left(G^{\mu v} \phi_{; \mu}^{\sigma} \phi_{; \nu}^{\rho}-m^{2} \phi^{\sigma} \phi^{\rho}\right) \sqrt{-G} \mathrm{~d}^{4} x,
$$

where the semicolon denotes the covariant derivative with respect to $G$.

Our construction produces the new Lagrangian $\tilde{\mathcal{L}}$ defined by

$$
\begin{aligned}
\tilde{\mathcal{L}}\left(j^{1} \phi, j^{2} \eta\right)= & \frac{1}{2} \eta^{c}{ }_{, \sigma} \eta^{d}{ }_{, \rho} g_{c d}\left(\kappa^{\mu}{ }_{a} \kappa^{\nu}{ }_{b} g^{a b}\left[\phi^{\sigma}{ }_{, \mu}+\left(\eta^{g}{ }_{, \mu \tau}+\eta_{, \mu}^{e} \eta^{f}{ }_{, \tau} \gamma_{e f}^{g}\right) \kappa^{\sigma}{ }_{g} \phi^{\tau}\right]\right. \\
& \left.\times\left[\phi^{\rho}{ }_{, v}+\left(\eta^{h}{ }_{, \nu \xi}+\eta^{p}{ }_{, \nu} \eta^{q}{ }_{, \xi} \gamma_{p q}^{h}\right) \kappa^{\rho}{ }_{h} \phi^{\xi}\right]-m^{2} \phi^{\sigma} \phi^{\rho}\right) \sqrt{-g}\left(\operatorname{det} \eta_{*}\right) \mathrm{d}^{4} x
\end{aligned}
$$

where $\eta_{*}$ is the Jacobian of $\eta$ and we have made use of (3.4).

Now we turn to the Euler-Lagrange equations for the $\eta^{a}$ which, since $\widetilde{\mathcal{L}}$ is second order in $\eta^{a}$, are

$$
\frac{\partial \widetilde{L}}{\partial \eta^{a}}-\frac{\partial}{\partial x^{\mu}}\left(\frac{\partial \widetilde{L}}{\partial \eta^{a}, \mu}\right)+\frac{\partial^{2}}{\partial x^{\nu} \partial x^{\mu}}\left(\frac{\partial \widetilde{L}}{\partial \eta^{a}, \mu \nu}\right)=0
$$

for $a=1, \ldots, \operatorname{dim} X$. The calculation of the lhs is similar to the previous one, but slightly more complicated. In any event, we find that $\eta$ satisfies the Euler-Lagrange equations $\Longleftrightarrow \nabla_{\mu} \mathfrak{T}^{\mu \nu}=0$, where now by the Hilbert formula

$$
\mathfrak{T}^{\mu \nu}=2 \frac{\delta L}{\delta G_{\mu \nu}}=2\left[\frac{\partial L}{\partial G_{\mu \nu}}-\frac{\partial}{\partial x^{\rho}}\left(\frac{\partial L}{\partial G_{\mu \nu, \rho}}\right)\right] .
$$

Thus for (first-order) derivative couplings the covariance field remains vacuously dynamic. It is likely that this will remain true for derivative couplings of arbitrary order, but we have not verified this as yet.

\section{Acknowledgments}

We dedicate this paper to Darryl Holm on his 60th birthday. We thank him for his interest in the ideas in this paper and for his many inspiring works over the years. MJG and JEM thank the National Science Foundation for its occasional support of work of this sort. MCL was partially supported by DGSIC (Spain) under grant MTM2007-60017.

\section{References}

Anderson J L 1967 Principles of Relativity Physics (New York: Academic)

Gotay M J and Marsden J E 1992 Stress-energy-momentum tensors and the Belinfante-Rosenfeld formula Contemp. Math. 132 367-92

Gotay M J and Marsden J E 2008a Momentum Maps and Classical Fields in preparation

Gotay M J and Marsden J E 2008b Parametrization theory, in preparation

Isham C and Kuchař K 1985 Representations of spacetime diffeomorphisms: I. Canonical parametrized field theories Ann. Phys., NY 164 288-315 
Kuchař K 1973 Canonical quantization of gravity Relativity, Astrophysics and Cosmology ed W Israel (Dordrecht: Reidel) pp 237-88

Marsden J E and Hughes T J R 1983 Mathematical Foundations of Elasticity (Englewood Cliffs, NJ: Prentice-Hall) Misner C W, Thorne K and Wheeler J A 1973 Gravitation (San Francisco: Freeman)

Post E J 2007 Formal Structure of Electromagnetics: General Covariance and Electromagnetics (New York: Dover) 